\title{
Antenatal Recognition of Megacystis Microcolon Intestinal Hypoperistalsis Syndrome (MMIHS): An Impact on Neonatal Prognosis
}

\begin{abstract}
Megacystis-microcolon-intestinal hypoperistalsis syndrome (MMIHS) syndrome is an autosomal recessive disorder (in some cases) characterized by a microcolon, a distended non-obstructed bladder, and decreased or absent intestinal peristalsis. MMIHS is not commonly diagnosed in the antenatal period due to its rarity and similarities with other more common antenatal conditions. Late diagnosis is associated with poorer prognosis. Prenatal diagnosis helps parents make decisions about their pregnancy guided by pediatric prenatal consultation regarding interventions and prognosis. Most medical and surgical interventions focus on complications of MMIHS after postnatal diagnosis. This case report discusses the prenatal diagnosis of MMIHS using serial ultrasonography and the early interventions designed foreseeable complications resulting in an extension of life beyond the historical limits.
\end{abstract}

Keywords: Berdon syndrome; Megacystis microcolon intestinal hypoperistalsis syndrome; Gastrointestinal motility; Intestinal pseudo-obstruction

Received: April 07, 2020; Accepted: May 14, 2020; Published: May 21, 2020

\section{Crystal Joseph ${ }^{1}$, Amartha Gore ${ }^{2 *}$, Shealy $\mathrm{O}^{\prime}$ Connell ${ }^{2}$ and Ronald Thomas ${ }^{2}$}

1 Department of Medicine, Drexel University College of Medicine, Philadelphia, PA, USA

2 Department of Obstetrics and Gynecology, Allegheny Health Network Consortium: Western Pennsylvania Hospital, Pittsburgh, PA, USA

\section{*Corresponding author: Amartha Gore ogburuog@gmail.com}

Department of Obstetrics and Gynecology, Allegheny Health Network Consortium: Western Pennsylvania Hospital, Pittsburgh, PA, USA.

Citation: Joseph C, Gore A, O'Connell S, Thomas R (2020) Antenatal Recognition of Megacystis Microcolon Intestinal Hypoperistalsis Syndrome (MMIHS): An Impact on Neonatal Prognosis. J Rare Disord Diagn Ther Vol.6 No.2:2

\section{Introduction}

Megacystis microcolon intestinal hypoperistalsis syndrome (MMIHS) (also known as the Berdon syndrome) is a rare congenital disorder characterized by a dilated non-obstructive urinary bladder and hypoperistalsis of the gastrointestinal tract. There is recognized strong female predilection. The disorder is thought to arise from defects primarily affecting the gastrointestinal and renal tracts. It is suggested that intestinal smooth muscle myopathy is responsible for the hypofunctioning intestine. Reduced actin level, degeneration of vacuoles and reduction in cytoskeleton protein has also been described. In the context of obstructive-like renal findings, the presence of normal to elevated amniotic fluid volumes may suggest the diagnosis. The overall prognosis is considered to be poor and treatment has been shown to be generally ineffective. It is usually lethal within the first year of life.

\section{Case Report}

A 30-year-old gravida 2 para 1 Caucasian female underwent a routine anatomy scan at 21 weeks and 5 days gestation demonstrating a female fetus with an enlarged fetal bladder measuring $4.1 \times 2.5 \times 2.8 \mathrm{~cm}$ ) depicted in Figure 1. There was suggestion of a keyhole appearance to the urethra. The stomach, ureters and kidneys were noted to be normal in appearance; the differential diagnosis was directed by a note of a urethral narrowing and the proposed leading diagnosis was atypical posterior urethral valve syndrome. The decision was made to complete follow-up sonograms every two to three weeks to monitor for additional change. A follow-up scan (Figure 2) at 24.4 weeks gestation demonstrated persistence in megacystis without emptying on exam, along with dilated ureters (3 $\mathrm{mm}$ ) and bilateral renal pyelectasis $(5 \mathrm{~mm})$. The amniotic fluid volume was normal; prune belly syndrome and lower urinary tract obstruction (LUTO) were added to the differential. At the 26 weeks and 4 days gestation scan, megacystis microcolon intestinal hypoperistalsis syndrome was highlighted as a possible etiology due to persistence of mild renal pyelectasis, normal amniotic fluid levels in the setting of an enlarged bladder in a female gender fetus. The diagnosis of MMIHS became concrete 


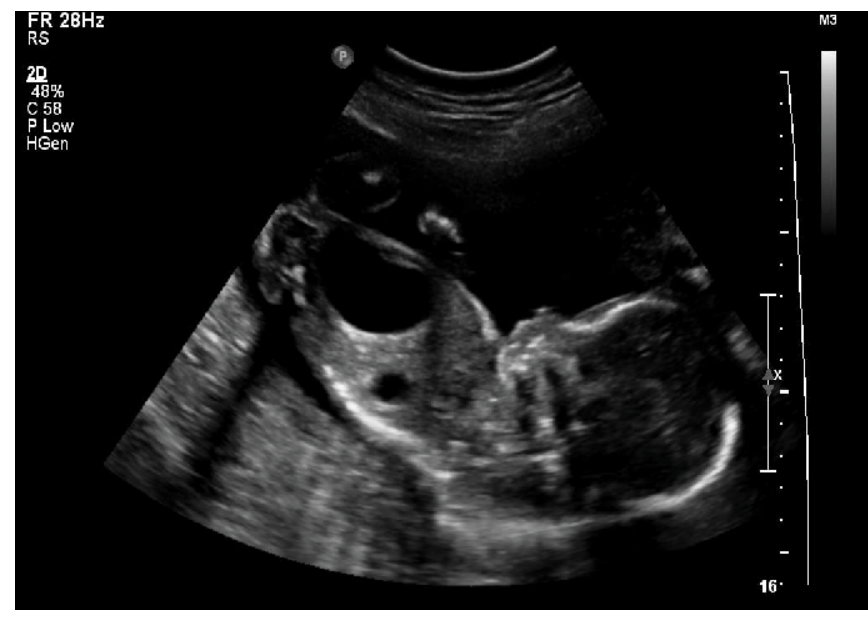

Figure 1 Prenatal ultrasound at 21.5 weeks gestation showing enlarged fetal bladder (measuring $4.1 \times 2.5 \times 2.8 \mathrm{~cm}$ ) with normal amniotic fluid around the fetus. There was suggestion of a keyhole appearance to the urethral side. The stomach is not enlarged. The specialist stated that at that time the ureters and kidneys are normal.

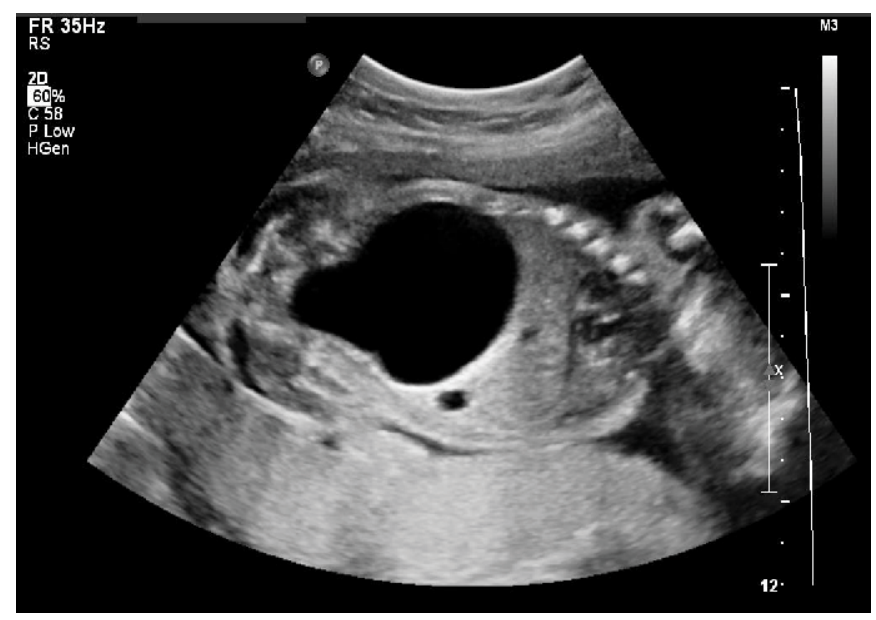

Figure 224.4 weeks gestation prenatal ultrasound. The specialist's dictation noted that the bladder measured $4 \times 6 \times 4 \mathrm{~cm}$ and did not empty during the exam. Normal amniotic fluid volume with both ureters visualized, measuring $3 \mathrm{~m}$ in caliber. Fetal renal pelvices are dilated, $\mathrm{R}>\mathrm{L}$.

with the 29-week scan (Figure 3): there was no sonographic evidence of an expanding colon, concerning for microcolon. A care team was established. The patient was connected with our NICU and was referred to a neonatal/pediatric gastroenterologist and pediatric urologists at the regional children's hospital. The pediatric gastroenterologist had a special interest in MMIHS and was at the time following five children with the diagnosis. The patient and her family were counseled and prepared with an outlined neonatal care plan. At 35 weeks 5 days gestation, the patient presented to Labor and Delivery with preterm premature rupture of membranes. She received a dose of betamethasone to

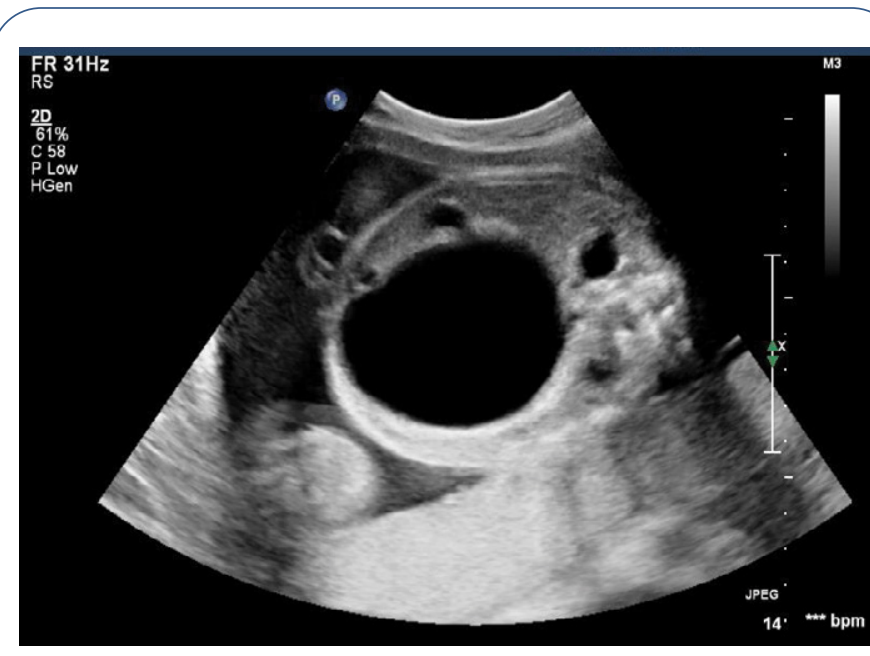

Figure 3 Prenatal ultrasound at 29.0 weeks gestation demonstrating the enlarged fetal bladder without evidence of a typical colon. The bladder dimensions were not available in this formal report.

aid with fetal lung function and bowel stabilization. She delivered a female infant, weighing 7 pounds, 10.8 ounces with Apgar scores of nine and nine at one and five minutes, respectively. The neonate was transferred as planned to the children's hospital for further management. Upon transfer, her diagnosis was confirmed postnatally with additional imaging, symptomatic therapy and rectal biopsy. Initial ultrasonography of the abdomen and pelvis demonstrated grade 3 hydronephrosis of the left kidney and over distended urinary bladder in the setting of neurogenic bladder. Upper GI study and follow-up abdominal Xray demonstrated markedly diminished bowel peristalsis and residual contrast in the small bowel consistent with hypomotility. The patient required a gastrostomy tube for gastric decompression and intermittent bladder catherization during her initial admission. Rectal biopsy was completed and demonstrated submucosal and myenteric ganglion cells with focal ulceration, acute and chronic transmural inflammation of the distal ileum, ruling out Hirschsprung disease and suggestive of diversion type colitis.

Microarray results demonstrated $\operatorname{Arr}(1-22, X) \times 2$, normal female. The childrens hospital did send ACTG2 sequencing; however, the results were not available for review due to restrictions in accessing patient's records at the outside hospital. This year the family will celebrate their daughter's fourth birthday. Her clinical course is not without its complications as she has required chronic TPN and multiple hospitalizations related to malnutrition and sepsis.

\section{Discussion}

Megacystis-microcolon-intestinal hypoperistalsis syndrome (MMIHS) is defined by "the presence of a massively distended urinary bladder without lower urinary tract obstruction, a dilated small bowel and microcolon without peristalsis", according to Evers et al. [1]. It was first described in five female neonates by Berdon et al. [2] and its presentation may also include a 
Table 1: Comparisons between MMIHS, LUTO and PBS.

\begin{tabular}{|c|c|c|c|}
\hline Variables & LUTO (PUV, Urethral Atresia & PBS & MMIHS \\
\hline $\begin{array}{l}\text { Timing of Initial Prenatal } \\
\text { Ultrasound } \\
\text { Findings }\end{array}$ & First Trimester & LUTO detected in Second Trimester & Second Trimester \\
\hline Dominant Gender & Males & Males & Female $=$ Males \\
\hline Bladder & $\begin{array}{l}\text { Thick Wall } \\
\text { Dilated }\end{array}$ & $\begin{array}{l}\text { Thin Wall } \\
\text { Dilated }\end{array}$ & $\begin{array}{l}\text { Thin Wall } \\
\text { Dilated }\end{array}$ \\
\hline Urethral Structure & Keyhole sign most often & Stricture entire length of urethra & None \\
\hline Amniotic Fluid & Oligohydramnios & Oligohydramnios & Normal or Polyhydramnios \\
\hline Bladder- Urethra Union & $\begin{array}{l}\text { Etiology Dependent } \\
\text { (PUV: Hypertrophied) }\end{array}$ & $\begin{array}{l}\text { Urethra Dilated (Wine glass or Funnel } \\
\text { shaped) }\end{array}$ & Normal Union \\
\hline Additional Findings & $\begin{array}{l}\text { 1. Urinary ascites } \\
\text { 2. Ureterectasis } \\
\text { 3. Caliectasis } \\
\text { 4. Subcortical cysts, small and } \\
\text { hyperechoic kidneys } \\
\text { 5. Ascites (in PUV) } \\
\text { 6. Thickened wall and } \\
\text { trabeculations (in PUV) } \\
\text { 7. Ureterectasis (in PUV) } \\
* * * \text { Etiology dependent }\end{array}$ & $\begin{array}{l}\text { 1. Triad: anterior abdominal } \\
\text { wall distension, megacystis, } \\
\text { pulmonary hypoplasia } \\
\text { 2. Dysplastic kidneys } \\
\text { 3. Hydronephrosis } \\
\text { 4. Ureterectasis } \\
\text { 5. Cryptorchidism }\end{array}$ & $\begin{array}{l}\text { 1. Dilated stomach } \\
\text { 2. Dilated bowel loops } \\
\text { 3. Bilateral hydronephrosis }\end{array}$ \\
\hline
\end{tabular}

megaesophagus and a meconium ileus [3]. MMIHS is caused by pathogenic variants in ACTG2 and is inherited in an autosomal dominant manner. The pathophysiology is associated with the loss of intestinal function related to smooth muscle myopathy. The neonate experiences reduced or absent bowel function, where enteral nutrition is not adequately completed. Without treatment, infants typically present with life-threatening sepsis, significant liver or renal failure or bowel obstruction.

\section{The challenges in diagnosis}

Diagnosis of MMIHS is usually made in the neonatal period due to anticipated complications related to bowel obstruction, bowel malabsorption and urinary retention. Most authors associate MMIHS with death before one year of life due to multiorgan failure and sepsis. Antenatal diagnosis is challenging due to its rarity and similarities with other conditions such as Prune Belly Syndrome (PBS), Lower Urinary Tract Obstruction (LUTO), and Posterior Urethral Valve (PUV) [4]. A comparison of these conditions is provided in Table 1. Visceral dysfunction is also a common finding in disorders such as Chronic Intestinal Pseudo-Obstruction (CIPO) and Multisystemic Smooth Muscle Dysfunction (MSMDS) [4].

\section{Implications of ultrasonography}

Although MMIHS is primarily diagnosed clinically, there are modalities that aid with diagnosis, which include ultrasonography, fetal MRI, amniotic fluid digestive enzyme assays and fetal urine analysis by vesicocentesis [5], abdominal radiography, upper gastrointestinal series, contrast enema and genomic sequencing (which ultimately confirms the diagnosis). The diagnosis is confirmed through genomic sequencing (whole exome sequencing or targeted sequencing) [6,7]. Ultrasonographic diagnosis has shown promise in improving overall outcomes by providing a means of detection of features in utero. In a 2014 MMIHS review of prenatal ultrasound findings, in $88 \%$ of cases had ultrasound findings leading to an eventual correct diagnosis of MMIHS including fetal megacystis with or without hydronephrosis; $10 \%$ showed isolated bilateral hydronephroses, and $2 \%$ showed an isolated dilated stomach [8]. Most initial ultrasound findings were reported in the second trimester. Multiple subsequent case reports have supported these ultrasound findings and their timeline of detection [7-9]. MMIHS is most commonly seen with normal or increased amniotic fluid volume; this was supported in the 2014 review (95\% of cases) and is collaborated by other case reports. Since MMIHS is frequently misdiagnosed as PBS, PUV, or LUTO, it is important to delineate the prenatal ultrasound findings to each of these syndromes (Table 1) [10-13].

Diagnosis in the antenatal period helps parents make informed decisions about their pregnancy and allows them to seek and coordinate early medical and surgical intervention in the postnatal period. Such interventions include use of prokinetic agents, chronic total parenteral nutrition, transabdominal urinary drainage and extensive abdominal surgery (including bowel or multivisceral transplantation or malrotation correction). Life expectancy seems to have improved with access to specialized care, innovations in parenteral nutrition and introduction of multivisceral transplantation; as the majority of patients with aggressive treatment will survive past 1 year [14].

\section{Conclusion}

Through this case, we have presented a scenario in which megacystis microcolon intestinal hypoperistalsis syndrome was diagnosed with serial fetal ultrasonography in the second and third trimesters. The sonographic features highlighted in this case include: an enlarged fetal bladder with persistently normal amniotic fluid volume, stable mild dilations in the ureters or kidneys and lastly, the lack of ultrasonographic evidence of an enhancing colon on the third trimester sonogram. The differential remained broad until evidence of microcolon was provided. 
This case highlights the impact of perinatal recognition and diagnosis on neonatal and pediatric outcomes, giving the patient access to an appropriate care team.

\section{References}

1 Evers J, Luigi R (2018) Megacystis-Microcolon-IntestinalHypoperistalsis Syndrome. In: Copel J, D'Alton M, Feltovich $\mathrm{H}_{\text {, }}$ Gratacos E, Odibo A, Platt L, Tutschek B (eds). Obstetric Imaging: Fetal Diagnosis and Care, Elsevier Health Sciences: Philadelphia, pp: 143-145.

2 Berdon WE, Baker DH, Blanc WA, Gay B, Santulli TV, et al. (1976) Megacystis-microcolon-intestinal hypoperistalsis syndrome: a new cause of intestinal obstruction in the newborn. Report of radiologic findings in five newborn girls. Am J Roentgenol 126: 957-964.

3 Al-Salem AH (2014) Megacystis microcolon intestinal hypoperistalsis syndrome: a report of a variant. Ann Pediatr Surg 10: 57-60.

4 Moreno CA, Metze K, Lomazi EA, Bertola DR, Barbosa RHA, et al. (2016) Visceral myopathy: clinical and molecular survey of a cohort of seven new patients and state of the art of overlapping phenotypes. Am J Med Genet 170: 2965-2974.

5 Garel C, Dreux S, Philippe-Chomette P, Vuillard E, Oury JF, et al. (2006) Contribution of fetal magnetic resonance imaging and amniotic fluid digestive enzyme assays to the evaluation of gastrointestinal tract abnormalities. Ultrasound Obstet Gynecol 28: 282-291.

6 Ambartsumyan L (2019) Megacystis-Microcolon-Intestinal Hypoperistalsis Syndrome Overview. In: Adam MP, Ardinger $\mathrm{HH}_{\text {, }}$ Pagon RA, Wallace SE, Bean LJH, et al(eds). GeneReviews. Seattle, WA:1993-2020.

7 Buinoiu N, Panaitescu A, Demetrian M, lonescu S, Peltecu G, et

\section{Conflicts of Interest}

There are no financial conflicts that exist that need to be reported.

al. (2018) Ultrasound prenatal diagnosis of typical megacystis, microcolon, intestinal hypoperistalsis syndrome. Clin Case Rep 6 : 855-858.

8 Tuzovic L, Anyane-Yeboa K, Mills A, Glassberg K, Miller R, et al. (2014) Megacystis-microcolon-intestinal hypoperistalsis syndrome: case report and review of prenatal ultrasonographic findings. Fetal Diagn Ther 36: 74-80.

9 Oka Y, Asabe K, Shirakusa T, Nabeshima K (2008) An antenatal appearance of megacystis-microcolon-intestinal hypoperistalsis syndrome. Turk J Pediatr 50: 269-274.

10 Adeb M, Anupindi S, Carr M, Darge K (2012) An unusual urinary tract presentation in a case of megacystis microcolon intestinal hypoperistalsis syndrome. J Radiol Case Rep. 6: 1-7.

11 Osborne NG, Bonilla-Musoles F, Machado LE, Raga F, Bonilla F, et al. (2011) Fetal megacystis: Differential diagnosis. J Ultrasound Med 30: 833-841.

12 Haeri S (2015) Fetal lower urinary tract obstruction (LUTO): A practical review for providers. Matern Health Neonatol Perinatol 1:26.

13 Papantoniou N, Papoutsis D, Daskalakis G, Chatzipapas I, Sindos $M$, et al. (2010) Prenatal diagnosis of prune-belly syndrome at 13 weeks gestation: Case report and review of literature. J Matern Fetal Neonatal Medm10: 1263-1267.

14 Chowdhary SK, Wilcox DT, Ransley PG (2003) Posterior urethral valves: Antenatal diagnosis and management. J Indian Assoc Pediatr Surg 8: 163-168. 